\title{
Chest wall stimulation in evaluation of patients with implanted ventricular-inhibited demand pacemakers
}

\author{
S. Serge Barold ${ }^{1}$, Giovanni A. Pupillo, John J. Gaidula, and Joseph W. Linhart \\ From the Department of Medicine, Highland Hospital and the University of Rochester School \\ of Medicine and Dentistry, Rochester, New York (Dr. Barold and Dr. Gaidula); and from the \\ Department of Physiology and Medicine, The University of Texas Medical School at San \\ Antonio, San Antonio, Texas, U.S.A.
}

Chest wall electrical stimuli, too weak to affect the heart, act as electrical signals to an implanted ventricular-inhibited (QRS blocking) demand pacemaker which interprets them as originating from the heart and consequently responds according to its specifications.

Rapid external stimulation permits diagnostic interpretation of the spontaneous electrocardiogram by completely inactivating the implanted pacemaker. The slow random delivery of external stimuli throughout the cardiac cycle delineates the pacemaker refractory period after the emission of a pacing stimulus and after the sensing of a spontaneous beat.

During apparent fixed-rate pacing the demand capability of the pacemaker may be easily seen by appropriately timed chest wall stimulation which induces the pacemaker to sense a spontaneous QRS complex.

This simple technique may reveal subtle changes in pacemaker performance, and contributes to the understanding of pacemaker arrhythmias.

Newer and sophisticated modes of electrical pacing of the heart have made the electrocardiographic evaluation of demand (standby) pacemaker performance increasingly complex (Castellanos et al., 1969; Rubin, Arbeit, and Gross, 1969; Spritzer et al., 1969). Ventricular demand pacemakers monitor the $R$ wave of the electrocardiogram and may be classified as ventricular-inhibited or QRS-blocking (Castellanos et al., 1969; Furman, Escher, and Solomon, 1967), and ventricular-triggered or QRS-stimulating types (Furman and Escher, 1968; Sowton, 1968; Castellanos et al., 1969). Several workers have advocated the application of low voltage external electrical stimuli to assess the function of ventriculartriggered demand pacemakers (Smyth, Bacos, and Keller, 1968; Castellanos and Lemberg, 1969; Furman et al., 1969; Smyth, 1969; Samet et al., 1969b). Chest wall electrical stimuli, too weak to affect the heart, act as

Received 9 March 1970.

1 Address for reprints: Dr. S. Serge Barold, Highland Hospital, South Avenue at Bellevue Drive, Rochester, New York 14620 , U.S.A. electrical signals to an implanted demand pacemaker which interprets them as originating from the heart and consequently responds according to its specifications. This simple technique may also provide information about the performance of ventricular-inhibited demand pacemakers, and permit analysis of the underlying electrocardiogram in patients constantly paced, by completely suppressing pacemaker discharge (Furman et al., 1969; Samet et al., 1969a; Sowton et al., 1969).

This report describes our experience with the chest wall stimulation technique in patients with ventricular-inhibited demand pacemakers.

\section{Methods}

Twenty patients with pervenous ventricularinhibited pacemakers of the Medtronic Demand type $^{2}$ also known as QRS blocking demand pacemakers and five patients with fixed rate (asynchronous) pervenous ventricular pacemakers were studied.

${ }^{2}$ Model 584I Demand Pulse Generator utilizing a bipolar catheter. Medtronic Inc., Minneapolis, Minnesota, U.S.A. 
Technique Control electrocardiograms, usually lead II or VI, are obtained before the procedure. Two small suction cup electrodes are then placed over the anterior chest of the patient usually where leads VI and V5 are recorded. Good contact of the electrodes is essential. Liberal use of conducting paste and the production of erythema by rubbing the skin facilitate the delivery of the stimuli by avoiding high skin resistance. The suction cups are then connected to a portable fixedrate pacemaker by means of standard alligator clip cables. External chest wall stimulation is initially applied at a rate Io to 20 beats a minute faster than the control rate. The response to stimulation is recorded, if possible, in a lead where the direction of the external stimuli is opposite to the direction of the implanted pacemaker impulses, so that the activity of the two pacemakers is readily distinguishable. The direction of the external stimuli in the electrocardiogram may be modified by either switching the connexions between the positive and negative poles of the external pacemaker or by selecting another lead or placement site for the suction electrodes. The amperage of the external pacemaker delivering the chest wall stimulation is gradually increased until a response occurs in the monitored electrocardiogram, or a maximum of $25 \mathrm{~mA}$ has been reached.

When chest wall stimuli are recorded on the electrocardiogram but evoke no response, inappropriate direction and amplitude of the chest wall stimuli must be excluded before considering the implanted pacemaker defective or non-responsive. The electrodes may have to be repositioned in many different areas until the optimal location is found, and occasionally only a change in polarity of the stimuli will achieve the required effect. In some patients the procedure requires considerable patience, to deliver a sufficient potential difference between the tip and proximal electrodes of the bipolar catheter. In difficult cases the cup electrodes are positioned along the direction of the mean frontal plane vector of the pacemaker impulse which can easily be calculated from the electrocardiogram. At times the entire chest wall, including the back of the neck and epigastrium, may have to be scanned to find the optimal delivery site.

Irregular pacemaker inactivation by chest wall stimulation simulating pacemaker malfunction may occur when one of the chest wall electrodes lies very near the optimal site for complete suppression (see Fig. 3, strip A). Shifting one of the electrodes a very short distance will then result in complete inactivation of the implanted pacemaker.

Though the patients appreciated chest wall stimuli of high amplitude, the discomfort was so slight that the procedure never had to be discontinued for that reason.

When the patient's spontaneous rate is faster than the present rate of the implanted pacemaker, and no pacemaker activity appears on the electrocardiogram, chest wall stimulation produces no effect. In this situation the pacing ability of the implanted pacemaker may be evaluated by converting it to a fixed rate unit, with the Model 5855 .

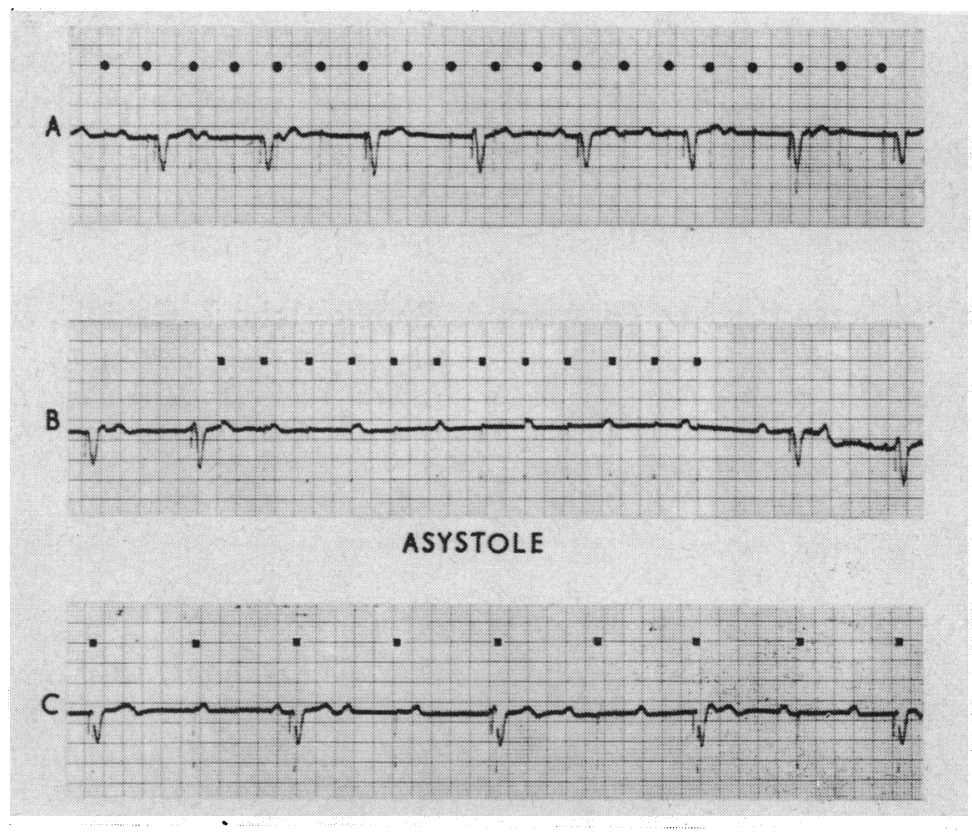

FIG. I Lead VI. Black dots depict external stimuli. Chest wall stimulation at $130 / \mathrm{min}$. completely suppresses the implanted pacemaker, while critically timed chest wall stimulation slightly faster than the automatic rate of the pacemaker induces 2:I pacemaker block rather than complete suppression (see text).

FIG. 2 Lead VI from same patient as in Fig. I. Black dots depict external stimuli. Determination of refractory period (R.P.) by chest wall stimulation (see text).

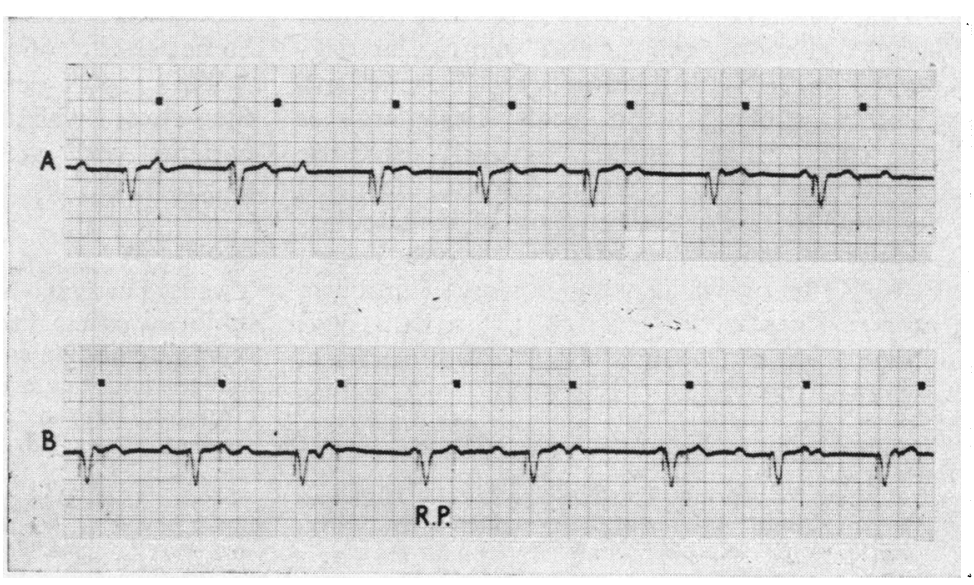


External rate control transmitter. If pacing occurs, carotid sinus pressure may be safely applied to slow the spontaneous rate, allowing escape of the pacemaker whose performance may then be assessed by chest wall stimulation.

Complete inactivation of the Medtronic demand pacemaker may be achieved by rapid chest wall stimulation. Accurate determination of the refractory period (interval following pacemaker spike when no external or intracardiac electrical activity deactivate the pacemaker) may be accomplished by the random delivery of external stimuli at a slow rate throughout the cardiac cycle and analysis of long electrocardiographic strips. The shortest interval between a sensed external stimulus and the preceding implanted pacemaker spike, or conversely the longest interval between an unsensed external stimulus and the preceding implanted pacemaker spike, indicates the pacemaker refractory period following its electrical discharge.

Three intervals are measured to evaluate the response to chest wall stimulation: $\mathrm{P}=$ implanted pacemaker spike; $E=$ external pacemaker spike chest wall stimulation; $Q=$ onset of spontaneous QRS complex.

a) Fixed rate (automatic) of the implanted pacemaker: PP interval.

b) Escape interval of the implanted pacemaker after an external stimulus: EP interval.

c) Escape interval of the implanted pacemaker after a spontaneous $Q R S$ complex: $Q P$ interval.

In our experience the $Q P$ and EP intervals, though different, usually remain constant for any particular patient provided the configuration of the $\mathrm{QRS}$ complex does not change and the energy of the external stimuli is above the threshold required to activate the sensing circuit of the pacemaker. For Medtronic implantable demand pacemakers $\mathrm{PP}>\mathrm{QP}>\mathrm{EP}$, since the abbreviation of the escape interval depends on QRS morphology (Spritzer et al., 1969). When an external stimulus above threshold falls up to 20 milliseconds outside the measured refractory period, the EP interval may be shorter than the EP interval from stimuli occurring later (see Fig. 2). Similarly, when the energy of the external stimuli is barely sufficient to inhibit the implanted pacemaker, 'partial sensing' with irregularly shortened EP intervals may occur.

\section{Results}

Chest wall stimulation successfully inactivated the 20 implanted Medtronic demand pacemakers while the five fixed rate pacemakers remained unaffected. Chest wall stimulation causes complete disappearance of the implanted pacemaker impulses, thereby allowing the emergence of the patient's spontaneous rhythm, if any. Extreme caution is required because prolonged asystole or marked bradycardia may be precipitated in some patients. Many patients, however, display spontaneous activity. The external chest wall stimuli do not usually interfere with the

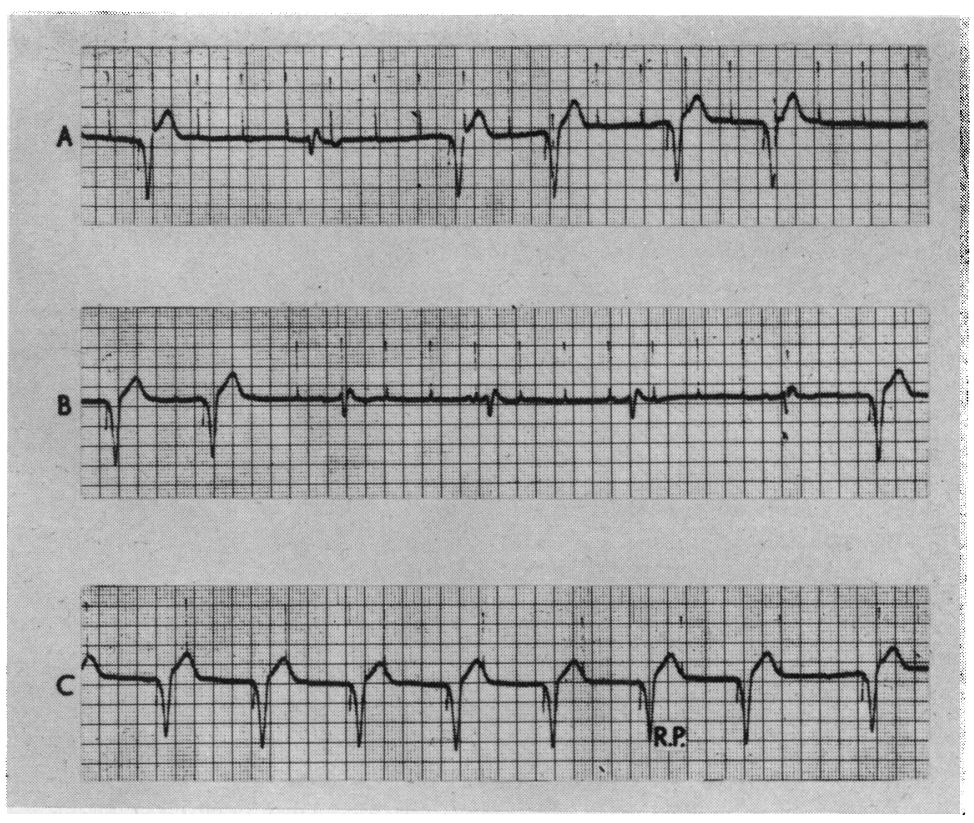

FIG. 3 Lead III showing pitfall of the chest wall stimulation technique by inappropriate placement of chest wall electrodes (see text).

FIG. 4 Lead VI shows how chest wall stimulation may be used to assess pacemaker characteristics, when the spontaneous rhythm is faster than the preset automatic pacemaker rate. $C S P=$ carotid sinus pressure (see text).

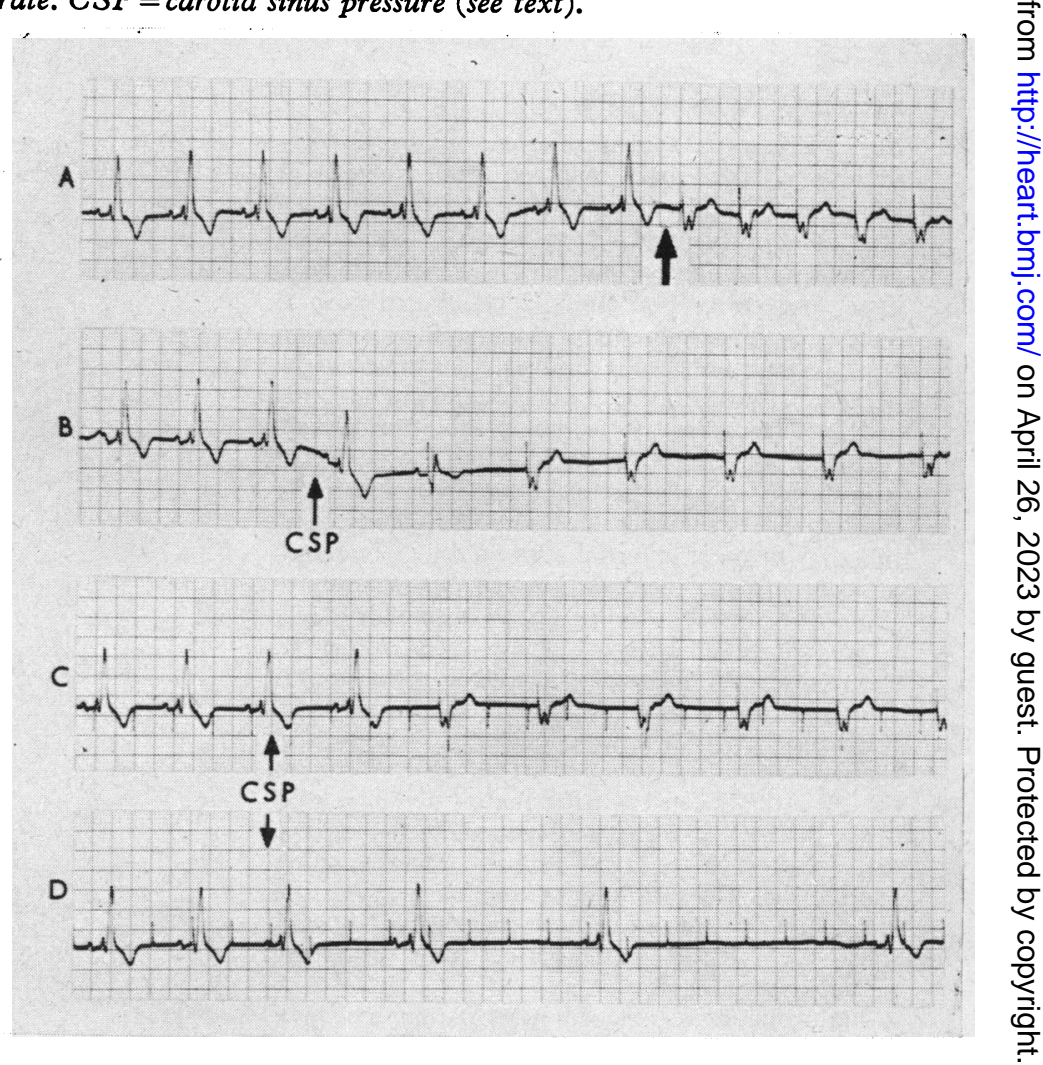


interpretation of the electrocardiogram, since they occur at random. Chest wall stimulation establishes the blocking property of the pacemaker, and permits, in the absence of severe bradycardia, the recording of an electrocardiogram for analysis and comparison with previous tracings. The electrical field generated by chest wall stimulation prevents the recording of $\mathrm{V}$ leads in the immediate vicinity of the stimulating chest electrodes. Often, a full I2-lead electrocardiogram may be obtained by repositioning the chest wall electrodes at another site, but this may require a lot of manipulation, and may not invariably succeed.

The refractory period of the Medtronic implantable demand pacemaker, as determined by chest wall stimulation, measured 380-440 msec. in all 20 cases.

A spontaneous QRS complex occurring substantially earlier than $380 \mathrm{msec}$. after a sensed external stimulus may be detected (see Fig. 6, strip D); the conversealso holds true when an external stimulus follows a sensed spontaneous QRS complex (see Fig. 5, strip C, Fig. 6, strip C). Though these observations have little clinical counterpart, they help characterize pacemaker behaviour, and indicate that in this particular type of pacemaker the sensing refractory period differs from the refractory period following the delivery of a pacing stimulus.

\section{Illustrative examples}

Fig. I illustrates the importance of proper positioning of the chest wall electrodes. Strip A shows regular Medtronic ventricular pacing at a rate of $55 / \mathrm{min}$. (PP=1100 msec.). Chest wall stimulation applied at a rate of 130 a minute produces no effect. The chest electrodes in strip B have been repositioned and chest wall stimulation at the same amplitude and rate suppresses the Medtronic pacemaker. The spontaneous electrocardiogram during chest wall stimulation reveals complete ventricular asystole. Strip $C$ shows that stimulation at a faster rate than the automatic rate of the pacemaker may not completely suppress its firing if external stimulation is inappropriately timed. Stimulation at 58 a minute ( $\mathrm{EE}=\mathrm{I0} 0 \mathrm{msec}$.) produces $2:$ I pacemaker block. Every alternate external spike falls about 50 msec. after the internal pacemaker spike and is therefore not sensed because it falls inside the refractory period of the pacemaker. The second external stimulus occurs just before the end of the PP interval, well outside the refractory period of the pacemaker which senses it, thereby blocking the delivery of the next impulse and recycling itself. Since the EP interval is shorter than the EE interval, the implanted pacemaker escapes after the second external stimulus and the sequence repeats itself.

Fig. 2 recorded from the same patient as Fig. I

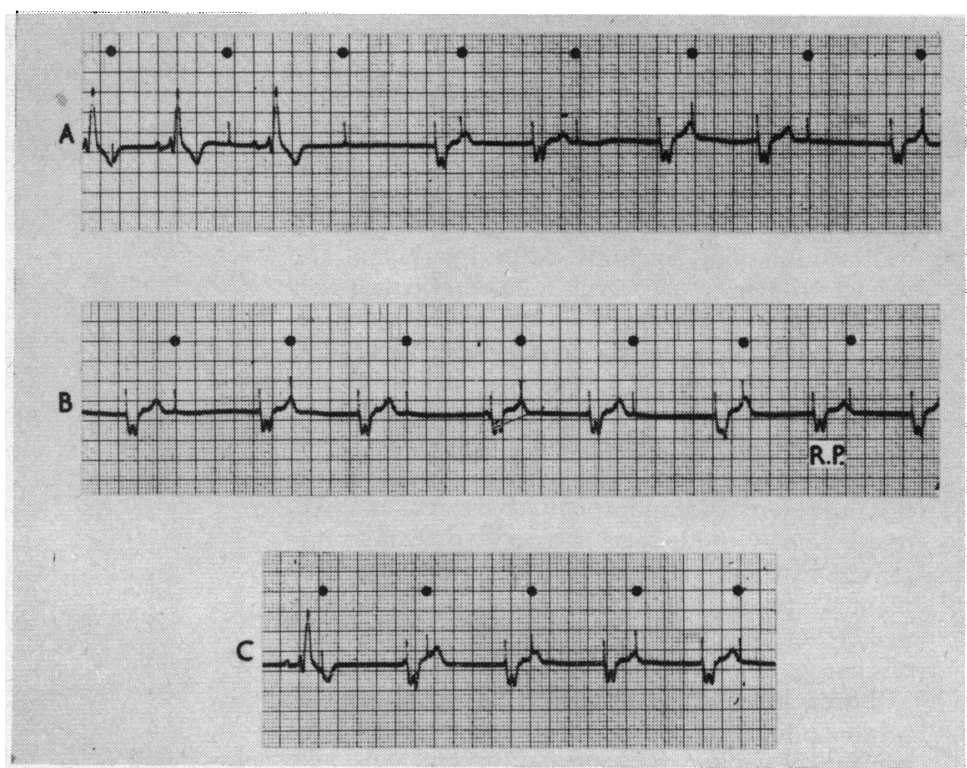

FIG. 5 Lead VI from the same patient as in Fig. 4 showing determination of the refractory period (R.P.). Black dots depict external stimuli (see text).

FIG. 6 Lead II showing relation of PP, EP, and QP intervals, and suppression of pacemaker by chest wall stimulation leading to the emergence of atrial fibrillation with a slow ventricular rate (see text).

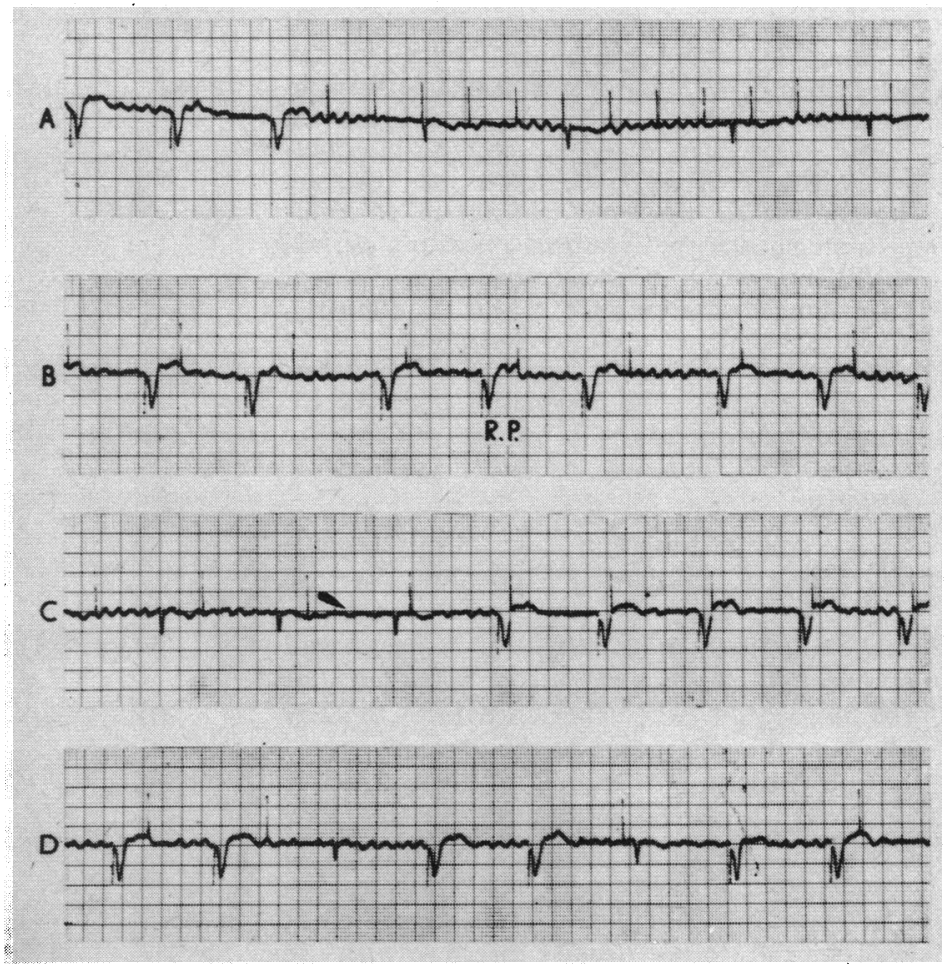


demonstrates the measurement of the pacemaker refractory period. External stimuli applied at a rate of 60 a minute occur at random in the cardiac cycle. Only the second and fifth external stimuli in strip $A$ and the third and fifth stimuli in strip $B$ are detected by the Medtronic pacemaker which recycles itself. The refractory period (R.P.) measures about $390 \mathrm{msec}$. as indicated in strip B by the longest interval between an implanted pacemaker spike and its succeeding unsensed external stimulus. The fifth external stimulus in strip A, occurring only $420 \mathrm{msec}$. after the internal spike, recycles the pacemaker. Note in strip $B$ that the third external stimulus, falling just outside the pacemaker refractory period, $420 \mathrm{msec}$. after the preceding implanted pacemaker spike, initiates an EP interval of $830 \mathrm{msec}$. in contrast to the fifth external stimulus which is followed by an EP interval of about $960 \mathrm{msec}$., similar to other EP intervals in Fig. I, strip C.

Fig. 3: external stimuli fall above the isoelectric line while implanted pacemaker stimuli fall below it. Erratic sensing of external stimuli during chest wall stimulation may occur when one of the chest wall electrodes lies very near the optimal site. This leads to irregular pacemaker inactivation simulating malfunction. Shifting one of the electrodes $0.8 \mathrm{~cm}$. in strip B induced complete pace- maker suppression and the emergence of sinus bradycardia. In strip $C$ the third last external stimulus falls just inside the R.P. (440 msec.) while the second last external stimulus falling $470 \mathrm{msec}$. after the implanted pacemaker spike recycles the pacemaker, indicating that the R.P. measures $440-470 \mathrm{msec}$.

Fig. 4: strip A shows that spontaneous rhythm is completely suppressing the implanted pacemaker. Application of the antenna from the external rate control transmitter directly over the implanted pacemaker (arrow) eliminates the sensing mechanism and confirms pacing capability. In strip B carotid sinus pressure demonstrates the demand function of the pacemaker during bradycardia. Chest wall stimulation at $25 \mathrm{~mA}$, with stimuli below the isoelectric line, does not inactivate the pacemaker during induced bradycardia. However, the mere change in polarity of chest wall stimulation in strip D successfully suppresses the implanted pacemaker during induced bradycardia.

Fig. 5 is recorded from the same patient as in Fig. 4. Carotid sinus pressure and chest wall stimulation at random permit measurement of the R.P. The last external stimulus in strip $B$ following the implanted pacemaker spike by $390 \mathrm{msec}$. fails to recycle the pacemaker which therefore

FIG. 7 Lead II showing how inactivation of ventricular pacemaker helps confirm the diagnosis of digitalis toxicity by revealing atrial flutter with $4: 1$ AV block and multifocal ventricular extrasystoles $(V)$. Black dots depict external stimuli (see text).

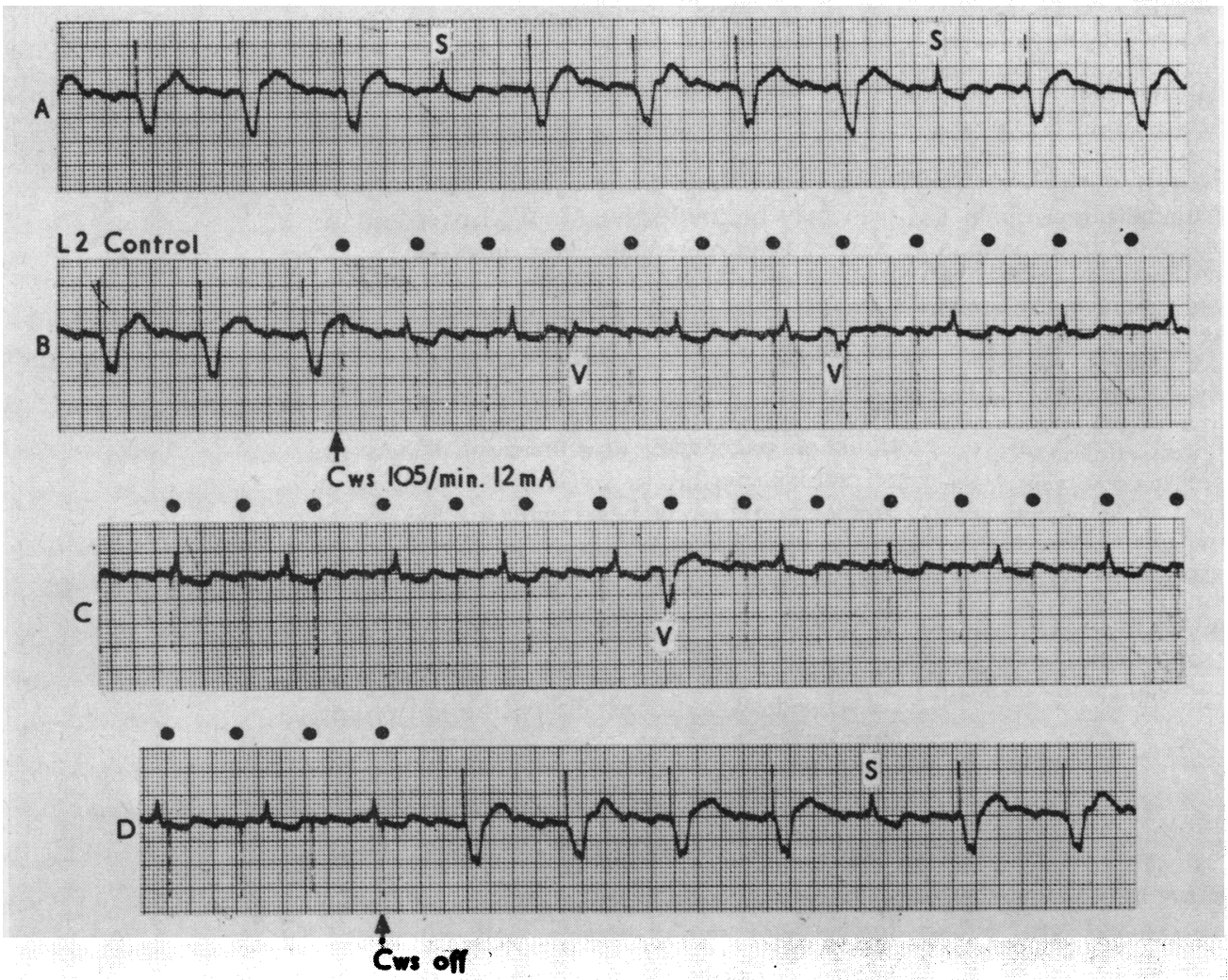


has a R.P. just over $390 \mathrm{msec}$. The first beat in strip $C$ is sensed by the pacemaker. An external stimulus applied 250 msec. after the onset of a spontaneous QRS is sensed by the pacemaker because the succeeding EP interval of $880 \mathrm{msec}$. equals the EP intervals in strips $A$ and $B$ when recycling occurs. If the first beat had been a paced beat, the external stimulus would not have been sensed.

Fig. 6: chest wall stimuli fall above the isoelectric line. Strip A shows the emergence of atrial fibrillation with a very slow ventricular response during stimulation. The R.P. of the implanted pacemaker approximates $370 \mathrm{msec}$. When sensing of external stimuli occurs the EP interval measures $900 \mathrm{msec}$. in contrast to the PP interval which measures ro40 msec. Sensing of the first three spontaneous beats and first four external stimuli occurs in strip C. The EP interval after the fourth external stimulus measures $900 \mathrm{msec}$., as in strip B when recycling occurs. Therefore the fourth external stimulus falling about $180 \mathrm{msec}$. after the onset of the third QRS complex must have been sensed by the pacemaker. Strip D shows early sensing of a spontaneous QRS complex after sensed external stimulus. The escape interval of the pacemaker following a regular spontaneous beat ( $3 \mathrm{rd}$ beat) measures $980 \mathrm{msec}$. The sixth beat, a spontaneous QRS, whose initial $\mathbf{R}$ wave is masked by a fibrillation wave, occurs about 100 msec. after the external stimulus. The QP interval following that beat approximates $980 \mathrm{msec}$, indicating that both the external stimulus and spontaneous QRS were sensed. Since premature beats cannot occur so early, these observations are of little clinical importance, but are, nevertheless, interesting. For the pacemaker in this patient, PP: $1040 \mathrm{msec}$. $\mathrm{QP}: 980$ msec. $>$ EP 900 msec.

Fig. 7 shows how chest wall stimulation helped confirm the diagnosis of digitalis toxicity in a patient with gastrointestinal symptoms. Strip A shows regular Medtronic demand ventricular pacing interrupted by two spontaneous sensed beats $(S)$ and an undulating baseline suggestive of atrial flutter. External stimulation applied at arrow in strip B completely inactivates the pacemaker, revealing atrial flutter with 4:I AV block and multifocal ventricular extrasystoles (V). After stopping digitalis for several days no ventricular extrasystoles were observed when chest wall stimulation was repeated, suggesting that digitalis intoxication had been responsible for the enhanced ventricular automaticity which incidentally was completely suppressed by ventricular pacing faster than the spontaneous rate by overdriving (Sowton, Leatham, and Carson, I964).

\section{Discussion}

When the spontaneous rhythm is faster than the preset escape interval of an implanted demand pacemaker, pacing ability may be evaluated by conversion to a fixed rate pacemaker. The Medtronic implanted demand pacemaker may thus be modified by an AC powered transmitter and antenna applied on the skin directed over the pacemaker. Setting the rate control of the transmitter Io to 20 beats above the spontaneous rhythm often avoids competitive rhythms (Fig. 4, strip A). Once the pacing potential of the pacemaker under study has been established, it may be challenged during spontaneous rhythm by the induction of bradycardia by vagal stimulating manoeuvres (Castellanos et al., 1968) or the administration of vagomimetic drugs (Sowton, 1967). When the pacemaker continuously activates the heart, the blocking behaviour of the unit may be assessed by accelerating the spontaneous rhythm with isoprenaline (Castellanos et al., I968), atropine, or exercise, but these procedures have certain inherent limitations and risks. Though useful, alterations in heart rate yield limited information regarding the sensing function of ventricular-inhibited pacemakers.

Chest wall stimulation offers a simple, safe, and effective method of evaluating some of the characteristics of ventricular-inhibited pacemakers. In general, demand pacemakers utilizing bipolar systems contain less efficient sensing mechanisms than unipolar systems because they register the difference in electric potential between two electrodes in close proximity. Whereas unipolar systems readily detect chest wall stimulation, bipolar systems may require considerable manipulation of the chest electrodes to achieve sensing of external stimuli, and occasionally, especially in patients with barrel-shaped chest, chest wall stimulation may be ineffective (J. W. Lister and W. Keller, 1969, personal communication).

The procedure determines not only the sensing capabilities of the pacemaker but its refractory period following electrical discharge. A change in the refractory period might conceivably be one of the first indications of pacemaker dysfunction. When the refractory period is excessively prolonged, many spontaneous beats will not be sensed and pacemaker stimuli may be delivered into the vulnerable period (Friedberg, 1969). The detection of chest wall stimulation by a QRSblocking pacemaker constitutes no guarantee that the spontaneous QRS complex can also be sensed. When constant pacing is present, appropriately timed chest wall stimulation stopped before a spontaneous beat permits analysis of the pacemaker response to the patient's own intracardiac voltage (Fig. 6, strip D). In general, malfunction of the demand mechanism of a pacemaker is either due to poor signal delivery (low voltage bipolar electrogram) or to component failure. 
In the former, chest wall stimulation will suppress the pacemaker, while in the latter inactivation may not be achieved. Therefore, chest wall stimulation may reveal subtle but clinically important changes in ventricularinhibited pacemaker performance undetectable by other means, and since the results are so easily reproducible, comparisons of serial tracings enhance its diagnostic value.

External stimuli may completely suppress the discharge of ventricular-inhibited pacemakers when the rate of appropriately timed stimulation exceeds the automatic rate of the pacemaker (Furman et al., 1969; Samet et al., 1969a; Sowton et al., 1969). This permits the emergence of the patient's spontaneous electrocardiogram which in the absence of ventricular asystole or obvious bradycardia may be recorded for diagnostic interpretation. Because of the risk of asystole or severe bradycardia, extreme caution should be exercised when using this technique.

The knowledge that little or no spontaneous activity exists (Fig. I, strip B), as revealed by this technique in any patient with a ventricular-inhibited pacemaker exhibiting even the slightest degree of pacing failure, assumes great importance if fatal asystole is to be prevented. In addition, since the average life of currently available power units is about two years or less, prophylactic replacement should be seriously considered after about 20-22 months in those patients with inadequate spontaneous rates during pacemaker inactivation by chest wall stimulation.

Chest wall stimulation, by completely suppressing a ventricular-inhibited pacemaker, may disclose diagnostic morphological or rhythm changes in the spontaneous electrocardiogram. Fig. 7 illustrates how suppression of the pacemaker by this technique confirmed the clinical diagnosis of digitalis toxicity by the observation of multifocal ventricular premature beats which disappeared after digitalis was withheld. The unmasked electrocardiogram may also reveal important morphological changes, such as the $Q$ waves of myocardial infarction, but ST segment and $T$ wave changes should be interpreted with some reservation since they may occur as a result of pacing per se (Chatterjee et al., 1969).

\section{References}

Castellanos, A., Jr., and Lemberg, L. (1969). Electrophysiology of Pacing and Cardioversion, p. 85. Appleton-Century-Crofts, Meredith Corporation, New York.

— - Jude, J. R., Mobin-Udden, K., and Berkovits, B. V. (1968). Implantable demand pacemaker. British Heart fournal, 30, 29.

—, Maytin, O., Lemberg, L., and Berkovits, B. V. (1969). Ventricular-triggered pacemaker arrhythmias. British Heart fournal, 31, 546.

Chatteriee, K., Harris, A. M., Davies, J. G., and Leatham, A. (1969). T-wave changes after artificial pacing. Lancet, $\mathbf{1}, 759$.

Friedberg, H. D. (1969). Syncope during standby cardiac pacing. British Heart fournal, 31, 281.

Furman, S., and Escher, D. J. W. (1968). Ventricular synchronous and demand pacing. American Heart fournal, 76, 445 .

- _ - Parker, B., and Solomon, N. (1969). Electronic analysis for pacemaker failure. Annals of Thoracic Surgery, 8, 57.

,-- , and Solomon, N. (1967). Standby pacing for multiple cardiac arrhythmias. Annals of Thoracic Surgery, 3, 327.

Rubin, I. L., Arbeit, S. R., and Gross, H. (1969). The electrocardiographic recognition of pacemaker function and failure. Annals of Internal Medicine, 7I, 603.

Samet, P., Center, S., Linhart, J. W., and Keller, W. (I969a). Selected current aspects of cardiac pacing. Electrocardiographic patterns. American fournal of Cardiology, 23, 702.

$\longrightarrow$, Hildner, F., Schoenfeld, C., and Narula, $O$. (I969b). Effect of chest wall stimulation on cardiac pacemaker function. Circulation, 39-40, Suppl. 3, I76.

Smyth, N. P. D. (1969). Cardiac pacemaking. Annals of Thoracic Surgery, 8, 166.

—, Bacos, J. M., and Keller, J. W. (1968). Progress in cardiovascular surgery. Experimental and clinical use of a variable parameter cardiac pacemaker. Diseases of the Chest, 53, 93.

Sowton, E. (1967). Cardiac pacemakers and pacing. Modern Concepts of Cardiovascular Disease, 36, 31.

- (1968). Ventricular-triggered pacemakers: clinical experience. British Heart fournal, 30, 363.

- , Balcon, R., Preston, T., Leaver, D., and Yacoub, M. (1969). Long-term control of intractable supraventricular tachycardia by ventricular pacing. British Heart fournal, 31, 700.

-, Leatham, A., and Carson, P. (1964). The suppression of arrhythmias by artificial pacemaking. Lancet, 2, 1098.

Spritzer, R. C., Donoso, E., Gadboys, H. L., and Friedberg, C. K. (1969). Arrhythmias induced by pacemaking on demand. American Heart fournal, 77, 6r9. 